\section{Disponibilidade de "açúcares de adição" no Brasil: distribuição, fontes alimentares e tendência temporal}

\author{
Availability of added sugars in Brazil: \\ distribution, food sources and time \\ trends
}

\section{Renata Bertazzi Levy',"1 \\ Rafael Moreira Claro" \\ Daniel Henrique Bandoni",III \\ Lenise Mondini'v}

\section{Carlos Augusto Monteiro"l,v}

'Departamento de Medicina Preventiva da Faculdade de Medicina da Universidade de São Paulo (USP).

"Núcleo de Pesquisas Epidemiológicas em Nutrição e Saúde da Universidade de São Paulo (NUPENS/USP).

II'Departamento de Saúde, Clínica e Instituições, Instituto de Saúde e Sociedade da UNIFESP.

IV Instituto de Economia Agrícola da Secretaria da Agricultura e Abastecimento do Estado de São Paulo.

` Departamento de Nutrição da Faculdade de Saúde Pública da Universidade de São Paulo (USP).

\section{Resumo}

Objetivos: Estimar o consumo de "açúcar de adição" pela população brasileira, nos estratos regionais e socioeconômicos, destacando suas principais fontes alimentares e verificar a tendência do seu consumo nas últimas décadas. Métodos: Contou-se com informações das Pesquisas de Orçamentos Familiares a partir da década de 80 sobre o tipo e a quantidade de alimentos e bebidas adquiridos pelas famílias brasileiras. Os indicadores analisados foram: \% das calorias de açúcar no total calórico da dieta e \% calórico das frações de açúcar de mesa e de açúcar adicionado aos alimentos pela indústria/kcal açúcar da dieta. Resultados: Em 2002/03, 16,7\% das calorias totais eram provenientes de "açúcar de adição" e sua participação mostrou-se elevada em todos os estratos regionais e de renda. A razão açúcar de mesa/açúcar adicionado pela indústria se inverte com o aumento da renda. A participação do açúcar de mesa nos últimos 15 anos foi reduzida, enquanto a contribuição do açúcar adicionado aos alimentos dobrou, especialmente por meio do consumo de refrigerantes e biscoitos. Conclusões: $O$ consumo de açúcar no Brasil excede largamente a recomendação da OMS e verificou-se importante alteração nas fontes de consumo.

Palavras-chave: Açúcar. Açúcar adicionado. Indústria do açúcar. Brasil. Pesquisa de Orçamentos Familiares. 


\section{Abstract}

Objective: To describe the regional and socio-economic distribution of consumption of added sugar in Brazil in 2002/03, particularly products, sources of sugar and trends in the past 15 years. Methods: The study used data from Household Budget Surveys since the 1980s about the type and quantity of food and beverages bought by Brazilian families. Different indicators were analyzed: \% of sugar calories over the total diet energy and caloric $\%$ of table sugar fractions and sugar added to processed food/ sugar calories of diet. Results: In 2002/03, of the total energy available for consumption, $16.7 \%$ came from added sugar in all regional and socio-economic strata. The table sugar/ sugar added to processed food ratio was inversely proportional to increase in income. Although this proportion fell in the past 15 years, sugar added to processed food doubled, especially in terms of consumption of soft drinks and cookies. Conclusions: Brazilians consume more sugar than the recommended levels determined by the WHO and the sources of consumption of sugar have changed significantly.

Keywords: Sugar. Add sugar. Sugar industry. Brazil. Household Budget Survey.

\section{Introdução}

Quimicamente, o termo "açúcares" refere-se a um grupo de compostos constituídos por átomos de carbono, hidrogênio e oxigênio e que se subdividem em monossacarídeos - como glicose, frutose e galactose - e dissacarídeos - como sacarose (glicose mais frutose) e lactose (glicose mais galactose $)^{1}$. Do ponto de vista do seu efeito sobre a saúde, importa destacar dois tipos de açúcares: aqueles encontrados naturalmente nos alimentos, como a frutose e a sacarose presentes nas frutas e a lactose presente no leite, e aqueles extraídos de alimentos (cana de açúcar, beterraba e milho) para posterior uso em preparações culinárias ou na elaboração de alimentos processados. A este último grupo de açúcares dá-se o nome de "açúcares de adição"'. Neste artigo usaremos a denominação "açúcares de adição".

Enquanto não há registro de malefícios à saúde decorrentes do consumo de açúcares naturalmente presentes nos alimentos, acumulam-se evidências de que a presença de "açúcares de adição" na dieta está associada ao aumento do risco de várias doenças, incluindo a cárie dental, a obesidade e outras doenças crônicas não transmissíveis. Por essa razão, as recomendações nutricionais da Organização Mundial da Saúde estipulam que o consumo de "açúcares de adição" não ultrapasse $10 \%$ do total de calorias da dieta. Os mecanismos que ligam o consumo de "açúcares de adição" a problemas de saúde são vários, incluindo a corrosão do esmalte dentário por ácidos resultantes do metabolismo dos açúcares por bactérias ${ }^{1,3}$, o comprometimento da auto-regulação do balanço energético (fome/saciedade), seja ele determinado pelo aumento da densidade energética da dieta ou pela ingestão de calorias na forma líquida ${ }^{1,4-8}$, e o aumento da concentração de triglicerídeos e diminuição da concentração da lipoproteína de alta densida$\mathrm{de}^{9,10}$. Além disso, o consumo elevado de "açúcares de adição" pode ser associado a uma diminuição no teor de proteínas e de micro-nutrientes na dieta ${ }^{11-13}$. 
São escassas as informações disponíveis sobre o consumo de "açúcares de adição" pela população, particularmente em países em desenvolvimento. O presente estudo objetiva estimar o consumo de "açúcares de adição" no Brasil, descrever suas principais fontes na alimentação e sua distribuição regional e socioeconômica e, ainda, estabelecer sua evolução nas últimas décadas.

\section{Metodologia}

Os dados utilizados neste estudo são originários de Pesquisas de Orçamentos Familiares (POF) realizadas pelo Instituto Brasileiro de Geografia e Estatística (IBGE) nos períodos de março de 1987 a fevereiro de 1988, de outubro de 1995 a setembro de 1996, e de junho de 2002 a julho de 2003. Neste último inquérito contou-se com amostra probabilística de todo território nacional, com 48.470 domicílios estudados (sendo 13.848 deles localizados nas áreas metropolitanas). Nos inquéritos anteriores, as amostras probabilísticas referem-se ao conjunto de domicílios das regiões metropolitanas do país (Belém, Fortaleza, Recife, Salvador, Belo Horizonte, Rio de Janeiro, São Paulo, Curitiba, Porto Alegre, Goiânia e Brasília), com 13.611 domicílios estudados em 1987/ 88 e 16.014 em 1995/96. Os procedimentos amostrais complexos utilizados nas pesquisas foram descritos em detalhes em publicações específicas ${ }^{14-16}$.

As informações referentes aos alimentos adquiridos em cada domicílio foram obtidas utilizando-se a caderneta de despesa coletiva da pesquisa. Nesta caderneta foram registradas todas as aquisições de alimentos feitas durante um período de sete dias consecutivos, incluindo quantidade, unidade de medida com seu equivalente em peso ou volume, valor da despesa, local de compra e a forma de obtenção do alimento.

Na presente investigação optou-se por adotar conjuntos de domicílios como unidade de estudo, considerando que a caracterização adequada do padrão de aquisição de alimentos em cada unidade domiciliar estudada poderia estar comprometida em vista do reduzido número de dias (período de sete dias) utilizado para a coleta de informações sobre aquisição de alimentos em cada domicílio.

Para a descrição do padrão de consumo de açúcar em 2002/03 foram utilizados agregados de domicílios homogêneos quanto ao nível socioeconômico e localização geográfica, reunidos em 443 estratos (definidos no plano amostral original da pesquisa). $\mathrm{O}$ número médio de domicílios estudado em cada um dos 443 estratos da POF 2002/03 foi 109,6 (variando de 9 a 804).

Para as análises de tendência de consumo do açúcar, nas áreas metropolitanas, fez-se necessário a utilização de informações do "Sistema IBGE de Recuperação Automática (SIDRA)*, uma vez que as quantidades adquiridas em gramas de cada produto em cada domicílio não estão disponíveis para as pesquisas de 1987/88 e 1995/96. O SIDRA disponibiliza informações detalhadas sobre a aquisição de alimentos e bebidas, segundo agregados de domicílios correspondentes a 10 intervalos de renda em cada uma das 11 áreas pesquisadas, totalizando, portanto, 110 agregados de domicílio para cada um dos três períodos estudados (1987/88, 1995/96 e 2002/03). O número médio de domicílios estudados em cada uma das 110 unidades originadas das amostras das áreas metropolitanas foi 123 em 1987/88 (variando de 45 a 351), 146 em 1995/96 (variando de 47 a 474) e 65,7 em 2002/03 (variando de 15 a 228). O fator de ponderação de cada unidade de estudo resultou sempre da somatória dos fatores amostrais de ponderação dos domicílios pertencentes a cada unidade em cada inquérito.

\section{Análise de dados}

Os indicadores construídos no presente estudo foram a participação percentual de "açúcares de adição" no total calórico da

* IBGE. SIDRA (Sistema IBGE de Recuperação Automática) Banco de dados agregados. Online. Disponível em http://www.sidra.ibge. gov.br. (Acessado em 22 março de 2010) 
aquisição de alimentos, e o percentual de participação das frações "açúcar refinado e outros adoçantes calóricos" e "açúcares adicionados pela indústria a alimentos processados".

Inicialmente, da quantidade bruta em gramas de cada alimento adquirido pelos domicílios excluiu-se, quando necessário, a fração não-comestível, aplicando-se para tanto fatores de correção recomendados pelo IBGE.** A quantidade comestível de cada alimento foi então convertida em calorias utilizando-se a Tabela Brasileira de Composição dos Alimentos - versão $1^{* * *}$ ou a tabela oficial de composição de alimentos dos Estados Unidos, versão $15^{* * * *}$, quando o alimento não estava disponível na primeira. A disponibilidade calórica diária per capita foi obtida convertendo-se os registros semanais das aquisições de alimentos de cada unidade de estudo em calorias, somando-se o total de registros e dividindo o resultado pela somatória de indivíduos em cada unidade de estudo. No caso específico da conversão para as calorias provenientes de "açúcares de adição", o cálculo envolveu duas etapas: a conversão de quantidades (em gramas) para calorias provenientes de carboidratos (usando, sempre que possível a tabela TACO) e a conversão das calorias provenientes de carboidratos para o correspondente calórico de "açúcares de adição" (usando a tabela oficial de composição de alimentos dos Estados Unidos, uma vez que a tabela brasileira não detalha os tipos de carboidratos de cada alimento).

Para o cômputo das calorias provenientes de "açúcares de adição" foram considerados tanto o "açúcar refinado e outros adoçantes calóricos" quanto o "açúcar adicionado aos alimentos processados pela indústria”. Outros adoçantes calóricos incluíram rapadura, melado, mel, glicose de milho e frutose. Os demais açúcares presentes naturalmente nos alimentos, como a frutose nas frutas e a lactose nos leites, foram considerados como parte do restante das calorias não provenientes de "açúcares de adição".

A partir dos dados da POF 2002/03, foram estimadas as médias da disponibilidade diária per capita de "açúcares de adição" (e respectivos intervalos de confiança) para o país como um todo e para as cinco macro-regiões geográficas, desagregadas em situação urbana ou rural, assim como para quintos da distribuição da renda per capita.

A participação percentual de alimentos selecionados na disponibilidade domiciliar total de "açúcares de adição" foi descrita para o país e segundo quintos da distribuição da renda per capita. Para esta finalidade, os alimentos adquiridos pelas famílias foram divididos em dois grupos fontes: "açúcar refinado (sacarose) e outros adoçantes calóricos"; e "açúcar adicionado aos alimentos processados pela indústria”.

A análise da tendência temporal dos indicadores de consumo de "açúcares de adição" foi realizada apenas para os domicílios estudados pelas POFs nas áreas metropolitanas do país, nos anos de 1986/87, 1995/96 e 2002/03.

Todos os procedimentos analíticos deste estudo foram executados com aplicativo Stata v.9.2, levando em conta o delineamento da amostra das POFs.

\section{Aspectos éticos}

O presente artigo não apresenta conflito de interesse real, potencial ou aparente por nenhum de seus autores e utilizou dados secundários, coletados pelo Instituto Brasileiro de Geografia e Estatística, disponíveis para consulta pública em meio eletrônico, dispensando a necessidade de submissão aos comitês de éticas conforme a resolução do CONEP.

*IBGE. Instituto Brasileiro de Geografia e Estatística. Estudo Nacional da Despesa Familiar - ENDEF 1974/75. Rio de Janeiro: IBGE; 1978.

*** NEPA/UNICAMP. Núcleo de Estudos e Pesquisas em Alimentação/Universidade Estadual de Campinas. Tabela Brasileira de Composição de Alimentos - TACO: Versão 1. Campinas, São Paulo: NEPA/UNICAMP; 2004.

****United States Department of Agriculture. Agricultural Research Service. USDA National Nutrient Database for Standard Reference. Release 15. Beltsville; 2002 


\section{Resultados}

A participação de "açúcares de adição" na disponibilidade domiciliar de alimentos no Brasil atingiu $16,7 \%$ do total de calorias, ultrapassando largamente o limite máximo de $10 \%$ recomendado pela OMS. Exceto na região Norte, onde os "açúcares de adição" corresponderam a $13 \%$ do total de calorias, nas demais regiões, a fração calórica dos "açúcares de adição ficou entre 16,3\% e $18,1 \%$. Não houve registro de diferenças substancias entre domicílios urbanos e rurais (Tabela 1 ).

A fração calórica de "açúcares de adição" foi elevada (sempre superior a 15\%) em todos os grupos de renda, não havendo um padrão de relação uniforme entre a participação de "açúcares de adição" e nível de renda dos domicílios (Tabela 2).

No conjunto dos domicílios brasileiros, três quartos das calorias oriundas de "açúcares de adição" provêm da fração "açúcares refinados e outros adoçantes calóricos"

Tabela 1 - Disponibilidade calórica total e proveniente de "açúcares de adição" nos domicílios brasileiros segundo região e situação urbana ou rural (2002/03).

Table 1 - Availability of total energy and energy from added sugars in Brazilian households by region and urban or rural status (2002/03).

\begin{tabular}{|c|c|c|c|c|c|c|c|c|c|}
\hline \multirow{2}{*}{$\begin{array}{l}\text { Região/situação } \\
\text { do domicílio }\end{array}$} & \multicolumn{3}{|c|}{$\begin{array}{l}\text { Disponibilidade total } \\
(\mathrm{kcal} / \mathrm{p} / \mathrm{dia})\end{array}$} & \multicolumn{3}{|c|}{$\begin{array}{l}\text { Disponibilidade de } \\
\text { "açúcares de adição" } \\
\text { (kcal/p/dia) }\end{array}$} & \multicolumn{3}{|c|}{$\begin{array}{c}\text { \% de participação de "açúcares } \\
\text { de adição"no total calórico } \\
\text { (\% kcal açúcar/ kcal total) }\end{array}$} \\
\hline & & \multicolumn{2}{|c|}{ IC 95\% } & Média & \multicolumn{2}{|c|}{ IC 95\% } & Média & \multicolumn{2}{|c|}{ IC 95\% } \\
\hline Urbana & $1.693,0$ & $1.572,6$ & $1.813,4$ & 208,5 & 191,1 & 225,9 & 13,3 & 12,4 & 11,1 \\
\hline Rural & $2.488,1$ & $2.327,3$ & $2.649,0$ & 283,7 & 236,2 & 331,3 & 13,6 & 11,5 & 9,5 \\
\hline Total & $1.882,8$ & $1.735,3$ & $2.030,3$ & 226,4 & 205,6 & 247,2 & 13,0 & 12,2 & 11,3 \\
\hline \multicolumn{10}{|l|}{ Nordeste } \\
\hline Urbana & $1.658,1$ & $1.604,5$ & $1.711,7$ & 260,4 & 246,4 & 274,5 & 16,4 & 15,7 & 15,1 \\
\hline Rural & $2.034,0$ & $1.931,0$ & $2.136,9$ & 320,2 & 297,4 & 343,1 & 17,0 & 15,9 & 14,8 \\
\hline Total & $1.757,0$ & $1.696,2$ & $1.817,8$ & 276,2 & 263,1 & 289,2 & 16,3 & 15,8 & 15,2 \\
\hline \multicolumn{10}{|l|}{ Sudeste } \\
\hline Urbana & $1.708,0$ & $1.585,9$ & $1.830,1$ & 297,0 & 263,2 & 330,9 & 18,1 & 17,1 & 16,1 \\
\hline Rural & $2.550,3$ & $1.934,3$ & $3.166,2$ & 437,2 & 349,5 & 525,0 & 19,4 & 17,6 & 15,9 \\
\hline Total & $1.775,6$ & $1.651,0$ & $1.900,2$ & 308,3 & 276,9 & 339,7 & 18,1 & 17,1 & 16,2 \\
\hline \multicolumn{10}{|l|}{ Sul } \\
\hline Urbana & $1.795,7$ & $1.666,1$ & $1.925,3$ & 281,3 & 261,1 & 301,5 & 16,5 & 15,7 & 14,9 \\
\hline Rural & $2.888,1$ & $2.417,9$ & $3.358,3$ & 434,9 & 361,6 & 508,2 & 16,9 & 15,4 & 13,8 \\
\hline Total & $1.973,6$ & $1.796,9$ & $2.150,2$ & 306,3 & 278,7 & 333,9 & 16,3 & 15,6 & 14,9 \\
\hline \multicolumn{10}{|l|}{ Centro-Oeste } \\
\hline Urbana & $1.607,9$ & $1.514,6$ & $1.707,3$ & 257,0 & 244,2 & 269,8 & 16,9 & 16,1 & 15,3 \\
\hline Rural & $2.521,3$ & $2.251,8$ & $2.790,8$ & 394,6 & 323,5 & 465,8 & 17,3 & 15,4 & 13,5 \\
\hline Total & $1.714,9$ & $1.596,7$ & $1.833,1$ & 273,1 & 253,9 & 292,4 & 16,7 & 16,0 & 15,3 \\
\hline \multicolumn{10}{|l|}{ Brasil } \\
\hline Urbana & $1.702,6$ & $1.637,2$ & $1.767,9$ & 278,4 & 259,6 & 297,2 & 16,8 & 16,2 & 15,6 \\
\hline Rural & $2.375,5$ & $2.171,7$ & $2.579,2$ & 368,0 & 335,2 & 400,8 & 16,5 & 15,8 & 15,0 \\
\hline Total & $1.805,2$ & $1.739,1$ & $1.871,2$ & 292,0 & 275,9 & 308,2 & 16,7 & 16,2 & 15,6 \\
\hline
\end{tabular}


Tabela 2 - Disponibilidade calórica total e proveniente de "açúcares de adição" nos domicílios brasileiros segundo quintos de renda per capita (2002/03).

Table 2 - Availability of total energy and energy from added sugars in Brazilian households by fifths of per capita income (2002/03).

\begin{tabular}{|c|c|c|c|c|c|c|c|c|c|}
\hline \multirow{3}{*}{$\begin{array}{l}\text { Quintos } \\
\text { de Renda } \\
1 \mathbf{1 0}^{\circ}\end{array}$} & \multicolumn{3}{|c|}{$\begin{array}{l}\text { "Açúcar de adição" } \\
\text { (kcal/p/dia) }\end{array}$} & \multicolumn{3}{|c|}{$\begin{array}{c}\text { "Açúcar de adição" } \\
\text { (\% kcal açúcar/ kcal total) }\end{array}$} & \multicolumn{3}{|c|}{$\begin{array}{c}\text { Calorias } \\
\text { (kcal/p/dia) }\end{array}$} \\
\hline & \multirow{2}{*}{$\frac{\text { Média }}{282,3}$} & \multicolumn{2}{|c|}{ IC 95\% } & \multirow{2}{*}{$\begin{array}{c}\text { Média } \\
15,4\end{array}$} & \multicolumn{2}{|c|}{ IC 95\% } & \multirow{2}{*}{$\begin{array}{c}\text { Média } \\
1.856,0\end{array}$} & \multicolumn{2}{|c|}{ IC 95\% } \\
\hline & & 264,7 & 299,9 & & 14,6 & 16,2 & & $1.754,9$ & $1.957,0$ \\
\hline $2^{\circ}$ & 305,7 & 270,0 & 341,8 & 16,1 & 15,1 & 17,1 & $1.899,9$ & $1.731,8$ & $2.068,0$ \\
\hline $3^{\circ}$ & 330,5 & 282,6 & 378,5 & 17,6 & 15,8 & 19,3 & $1.865,8$ & $1.687,2$ & $2.044,4$ \\
\hline $4^{\circ}$ & 266,3 & 233,7 & 298,8 & 15,8 & 15,1 & 16,5 & $1.676,8$ & $1.499,4$ & $1.854,2$ \\
\hline $5^{\circ}$ & 276,6 & 256,9 & 296,3 & 15,9 & 15,2 & 16,6 & $1.725,9$ & $1.637,3$ & $1.814,6$ \\
\hline
\end{tabular}

(sendo mínima a proporção dos outros adoçantes calóricos - menos de $0,6 \%$ do total de calorias). O quarto restante das calorias oriundas de "açúcares de adição" provém de alimentos processados, em particular de refrigerantes, doces, balas e chocolates e biscoitos (Tabela 3). A proporção dos "açúcares de adição" provenientes de alimentos processados aumentou com a renda domiciliar, aproximando-se da proporção proveniente dos "açúcares refinados e outros adoçantes calóricos" no quinto superior de renda ( $42,1 \%$ e $57,9 \%$, respectivamente) (Tabela 3).

Como já foi mencionado, a evolução da participação de "açúcares de adição" na disponibilidade domiciliar de alimentos no Brasil só pôde ser estudada nas áreas metropolitanas. As POFs realizadas nessas áreas metropolitanas em 1987/88, 1995/96 e 2002/03 evidenciam estabilidade na participação de "açúcares de adição": 15,9\%, 16,1\% e 16,3\%, respectivamente, (p de tendência 0,407). Entretanto, a fração de "açúcares de adição" oriunda de alimentos processados aumenta significativamente ao longo das três pesquisas, dobrando entre 1987/88 e 2002/03 (de 17,4\% para 35,5\%). Neste período a contribuição do refrigerante para o total de "açúcares de adição" aumenta em $200 \%$ (de $6,1 \%$ para $18,8 \%$ ) e a contribuição dos biscoitos aumenta em $100 \%$ (de 2,4\% para $5,2 \%)$. Os doces, balas e chocolates, apresentam aumento no período, porém não estatisticamente significativo.

Tabela 3 - Distribuição (\%) da disponibilidade de calorias oriundas de "açúcares de adição" nos domicílios brasileiros, por quintos de renda per capita, segundo alimentos fonte (2002/03).

Table 3 - Distribution (\%) of the availability of energy from added sugars in households, by fifths of per capita income, according to food source (2002/03).

\begin{tabular}{lcccccc}
\hline \multirow{2}{*}{ Alimentos fonte de açúcar } & \multirow{2}{*}{ Brasil } & \multicolumn{5}{c}{ Quintos de renda } \\
\cline { 3 - 7 } & & $1^{\circ}$ & $2^{\circ}$ & $3^{\circ}$ & $4^{\circ}$ & $5^{\circ}$ \\
\hline Açúcar refinado e outros adoçantes calóricos & 75,0 & $88,4^{* *}$ & 82,6 & 78,4 & 67,5 & 57,9 \\
Açúcares & 25,0 & $11,6^{* *}$ & 17,4 & 21,6 & 32,5 & 42,1 \\
$\quad$ em refrigerantes & 11,0 & $4,4^{* *}$ & 7,8 & 9,6 & 15,3 & 18,0 \\
$\quad$ em doces, balas e chocolates & 8,4 & $3,1^{* *}$ & 5,4 & 7,0 & 11,1 & 15,4 \\
$\quad$ em biscoitos & 4,2 & $3,5^{* *}$ & 3,4 & 4,0 & 4,4 & 5,5 \\
$\quad$ em outros alimentos* & 1,5 & $0,6^{* *}$ & 0,8 & 1,0 & 1,8 & 3,3 \\
\hline
\end{tabular}

* cereais matinais, bebidas lácteas, chás e sucos de frutas naturais adoçados / * sweetened breakfast cereals, milk beverages, teas and natural fruit juices.

** $\mathrm{p}$ significativo para tendência linear $/{ }^{* *}$ significant $p$ for linear trend 
Tabela 4 - Distribuição (\%) da disponibilidade de calorias oriundas de "açúcares de adição", segundo alimentos fontes. Áreas metropolitanas do Brasil (1987-2003).

Table 4 - Distribution (\%) of the availability of energy from added sugars according to food source. Metropolitan areas of Brazil (1987-2003).

\begin{tabular}{lccc}
\hline \multirow{2}{*}{ Alimento fonte } & \multicolumn{3}{c}{ Anos das Pesquisas } \\
\cline { 2 - 4 } & $1987 / 88$ & $1995 / 96$ & $2002 / 03$ \\
\hline Açúcar refinado e outros adoçantes calóricos & $82,6^{* *}$ & 78,9 & 64,5 \\
Açúcares & $17,4^{* *}$ & 21,1 & 35,5 \\
em refrigerantes & $6,1^{* *}$ & 10,0 & 18,8 \\
em doces, balas e chocolates & 8,3 & 7,1 & 10,1 \\
em biscoitos & $2,4^{* *}$ & 3,5 & 5,2 \\
em outros alimentos* & $0,6^{* *}$ & 0,5 & 1,4 \\
\hline
\end{tabular}

* cereais matinais, bebidas lácteas, chás e sucos de frutas naturais adoçados / * sweetened breakfast cereals, milk beverages, teas and natural fruit juices.

** p significativo para tendência linear / ** significant p for linear trend

\section{Discussão}

Os resultados do presente estudo revelaram consumo excessivo de "açúcares de adição" nos domicílios brasileiros, ultrapassando em mais de $60 \%$ o limite máximo de consumo recomendado pela OMS. Esse cenário foi observado em todas as regiões do país, nos meios urbano e rural e em todas as classes de renda. Nas áreas metropolitanas, a participação de "açúcares de adição" no total calórico da disponibilidade domiciliar de alimentos dos brasileiros manteve-se praticamente estável entre 1987/88 e 2002/03, embora tenha se registrado aumento substancial da fração proveniente de alimentos processados.

Evidências apontam para o aumento do consumo de "açúcares de adição" nas últimas décadas, tanto em países desenvolvidos quanto em alguns países em desenvolvimento, em parte pelas mudanças no padrão de vida decorrentes da urbanização e do aumento da renda. No período entre 1962 e 2000 o consumo per capita de "açúcares de adição" aumentou em aproximadamente $74 \mathrm{~g}$ diárias ${ }^{17}$.

Considerando o período analisado no presente estudo, entre 1987 e 2003, verificamos por meio de dados das Folhas de Balanço de Alimentos compiladas pela Organização das Nações Unidas para Agricultura e Alimentação - FAO, as quais expressam a quantidade média de alimentos disponível para consumo humano em cada país, que a participação do açúcar no total calórico per capita disponível nos países desenvolvidos pouco variou, a exemplo dos EUA (entre 16,5\% e 17,1\%) e dos países da União Européia (entre 10,9\% e 11,3\%), embora os patamares de consumo sejam bastante diferenciados. Nos países em desenvolvimento, como os da América Central e Caribe e Brasil, a variação de consumo no período ficou entre $15,1 \%$ e $18,1 \%$ e entre $13,2 \%$ e $17,6 \%$, respectivamente ${ }^{18}$.

A pequena variação no consumo de açúcar observada nos países mais ricos pode estar relacionada à saturação do mercado, à divulgação de informações que associam doenças ao consumo excessivo de açúcares e à reduzida elasticidade de preço de demanda nos países mais ricos ${ }^{19}$. Em 2005-2006 os dados da FAO indicavam que a disponibilidade de açúcar nos países desenvolvidos era cerca da metade daquela verificada nos países em desenvolvimento ${ }^{18}$.

O presente estudo tem o mérito de identificar, ao longo do tempo, mudanças importantes na participação do açúcar refinado e outros adoçantes calóricos, e do açúcar adicionado aos alimentos processados, sendo que o predomínio do primeiro sobre o segundo foi bastante atenuado no referido período (4,7 para 1,8 vezes), e a fração do açúcar proveniente de refrigerantes, 
doces, balas, chocolates e biscoitos, que representava aproximadamente $17 \%$ do total de açúcar da dieta ao final da década de 80 , duplicou (35\%) em 2002/03.

Embora não se conte com informações na literatura que permitam comparar as mudanças da participação do açúcar adicionado a alimentos processados, produto a produto, ao longo de décadas, o cenário que se apresenta no Brasil, ainda que o açúcar de mesa seja a principal fonte de consumo de açúcar, parece se assemelhar ao de países como os EUA, onde, em 35 anos, a participação de outros tipos de açúcar utilizados especificamente na confecção de alimentos processados cresceu largamente em detrimento do açúcar refinado ${ }^{20,21}$.

No Brasil a elevação da contribuição dos alimentos processados com o aumento do nível de renda (variando entre 11,6\% e 42,2\% entre os quintos extremos da distribuição de renda) pode ser um indicativo de mudanças nas fontes de consumo de açúcares, uma vez que no período entre 2003 e 2008 houve um aumento médio de $28,2 \%$ na renda média do brasileiro ${ }^{22,23}$.

De fato, merece atenção especial a observação do vertiginoso crescimento de refrigerantes, uma vez que o consumo elevado dessas bebidas está associado a uma menor qualidade da dieta, e a maiores riscos de desenvolvimento de doenças crônicas não transmissíveis, como a diabetes tipo $2^{24-27}$. Evidências sugerem ainda que as calorias consumidas através de meio líquido têm um menor poder de saciedade quando comparadas àquelas ingeridas através de alimentos sólidos ${ }^{28,29}$, resultando em desequilíbrio dos mecanismos orgânicos de autorregulação do consumo de alimentos $\mathrm{e}$, consequentemente, no ganho excessivo de peso.

Embora o aumento da participação de alimentos industrializados no consumo de açúcar não tenha se refletido no aumento do açúcar total da dieta, evidências associam o consumo de açúcar oriundo desta fonte à maior participação de gorduras e de ácidos graxos saturados e menor participação de carboidratos, que não o açúcar, na alimentação, enquanto nenhuma associação foi encontrada para o açúcar de mesa ${ }^{30}$.

Os resultados do presente estudo se referem apenas à aquisição de alimentos e bebidas para consumo no domicilio. No Brasil, os alimentos e bebidas adquiridos para consumo no domicílio respondem por aproximadamente $78 \%$ das despesas totais com alimentação das famílias em 2002/03 ${ }^{31}$. Assumindo-se que o preço por caloria dos alimentos adquiridos para consumo fora de casa seja igual ou superior ao preço dos alimentos adquiridos para consumo no domicilio, nossos resultados se aplicariam, pelo menos, a 3/4 do total de alimentos adquiridos.

O curto período (uma semana) de referência para a coleta de dados sobre as aquisições de alimentos feitas por cada domicílio nas POFs poderia, igualmente, constituir uma importante limitação deste estudo. No sentido de minimizar este efeito, adotamos como unidade de análise grupos de domicílios homogêneos quanto à localização territorial e características socioeconômicas, estudados ao longo de um período de 12 meses.

Apesar destas limitações, estudos comparando dados de pesquisas de orçamentos familiares com aqueles obtidos em inquéritos individuais de consumo apontam para considerável concordância entre os métodos $^{32,33}$. No caso específico do consumo de açúcar, um estudo realizado em quatro países europeus estimou coeficiente de correlação entre consumo de açúcar mensurado por pesquisa de orçamentos familiares e por inquérito individual de consumo em $0,74^{32}$. Por fim, dados de disponibilidade domiciliar de açúcares são, por vezes, considerados como uma boa aproximação do consumo deste nutriente, uma vez que as Pesquisas de Orçamentos Familiares podem refletir com grande acurácia o consumo real dos indivíduos no caso de alimentos utilizados como ingredientes de preparações, provavelmente devido à dificuldade dos indivíduos em relatar a quantidade consumida de um alimento ingerido como parte de uma preparação em inquéritos tradicionais de consumo ${ }^{30}$. 
O elevado consumo de "açúcares de adição" na população brasileira, verificado em todos os cenários estudados, excede largamente as recomendações internacionais, destacando a necessidade de intervenções visando a sua redução. Considerando que o açúcar proveniente de alimentos processados vem substituindo o açúcar de mesa como principal fonte de consumo dos açúcares nas últimas décadas, intervenções para reduzir o consumo de alimentos processados e incentivo a práticas alimentares saudáveis assumem caráter ainda mais emergencial.

\section{Referências}

1. Food and Agriculture Organization/World Health Organization. Carbohydrates in Human Nutrition. Geneva; 1998. (Report of a Joint FAO/WHO Expert Consultation).

2. Institute of Medicine. Dietary reference intakes for energy, carbohydrate, fiber, fat, fatty acids, cholesterol, protein, and amino acids. Washington: National Academy Press; 2002.

3. Touger-Decker R, Touger CL. Sugars and dental caries. Am J Clin Nutr 2003; 78: 881S-2.

4. Stubbs J, Ferres S, Horgan G. Energy density of foods: effects on energy intake. Crit Rev Food Sci Nutr 2000; 40: 481-515.

5. DiMeglio DP, Mattes RD. Liquid versus solid carbohydrate: effects on food intake and body weight. Int J Obes Relat Metab Disord 2000; 24: 794-800

6. Ludwig DS, Peterson KE, Gortmaker SL. Relation between consumption of sugar-sweetened drinks and childhood obesity: a prospective, observational analysis. Lancet 2001; 357: 505-8.

7. Anderson GH, Woodend D. Effect of glycemic carbohydrates on short-term satiety and food intake. Nutr Rev 2003; 61: S17-26.

8. Van Wymelbeke V, Thérond MEB, La Guéronnière V, Fantino M. Influence of repeated consumption of beverages containing sucrose or intense sweeteners on food intake. Eur J Clin Nutr 2004; 58: 154-61.

9. Fried SK, Rao SP. Sugars, hypertriglyceridemia, and cardiovascular disease. Am J Clin Nutr 2003; 78: 873S-80.

10. Kelly SAM, Moynihan PJ, Rugg-Gunn AJ, Summerbell CD. Review of methods used to estimate non-milk extrinsic sugars. J Hum Nutr Diet 2003; 16: 27-38.

11. Farris RP, Nicklas TA, Myers L, Berenson GS. nutrient intake and food group consumption of 10-year-olds by sugar intake level: the Bogalusa heart study. J Am College Nutr 1998; 17: 579-85.

12. Kranz S, Smiciklas-Wright H, Siega-Riz AM, Mitchell D. Adverse effect of high added sugar consumption on dietary intake in American preschoolers. J Pediatr 2005; 146: 105-11.
13. Alexy U., Sichert-Hellert W, Kersting M. Fortification masks nutrient dilution due to added sugars in the diet of children and adolescents. J Nutr 2002; 132: 2785-91.

14. Instituto Brasileiro de Geografia e Estatística. Pesquisa de orçamentos familiares, 1987/88: regiões metropolitanas. Rio de Janeiro: IBGE; 1991.

15. Instituto Brasileiro de Geografia e Estatística. Pesquisa dos orçamentos familiares 1995-1996: primeiros resultados. Rio de Janeiro: IBGE; 1997.

16. Instituto Brasileiro de Geografia e Estatística. Pesquisa de Orçamentos Familiares 2002-2003: análise da disponibilidade domiciliar de alimentos e do estado nutricional no Brasil. Rio de Janeiro: IBGE; 2004.

17. Popkin BM, Nielsen SJ. The sweetening of the world's diet. Obes Res 2003; 11: 1325-32.

18. Food and Agriculture Organization Statistics Database. FAO Statistics database. Rome: Food and Agriculture Organization. Disponível em http://faostat.fao.org/ faostat/collections? version $=$ ext \&hasbulk $=0$ [Acessado em dezembro de 2010]

19. Food and Agriculture Organization. Food outlookglobal market analysis. June, 2007. p. 22-24.

20. Beghin, JC, Jensen HH. Farm policies and added sugars in US diets. Food Policy 2008; 33: 480-8.

21. Wellss HF, Buzby JC. Dietary assessment of major trends in U.S. food consumption, 1970-2005. Economic information Bulletin 2008; 33: 17-18.

22. Instituto de Pesquisa Econômica Aplicada. IPEA DATA. Rio de Janeiro: Instituto de Pesquisa Econômica Aplicada. Disponível em http://www.ipeadata.gov.br/ [Acessado em novembro de 2010]

23. Neri MC, coordenador. Miséria, desigualdade e políticas de renda: o Real do Lula. Rio de Janeiro: FGV/IBRE/CPS; 2007.

24. Schulze MB, Manson JE, Ludwig DS. Sugar-sweetened beverages, weight gain, and incidence of type 2 diabetes in young and middle-aged women. JAMA 2004; 292: $927-$ 34. 
25. Vartanian LR, Schwartz MB, Brownell KD. Effects of soft drink consumption on nutrition and health: a systematic review and meta-analysis. Am J Public Health 2007; 97: 667-75.

26. Lim S, Zoellner JM, Lee JM, Burt BA, Sandretto AM, Sohn W et al. Obesity and sugar-sweetened beverages in African-American preschool children: a longitudinal study. Obesity 2009; 17: 1262-8.

27. Mueller NT, Odegaard A, Anderson K, Yuan JM, Gross M, Koh WP, Pereira M. Soft drink and juice consumption and risk of pancreatic cancer: The Singapore Chinese Health Study. Cancer Epidemiol Biomarkers Prev 2010; 19: 447-55.

28. Mourao DM, Bressan J, Campbell WW, Mattes RD. Effects of food form on appetite and energy intake in lean and obese young adults. Int J Obes 2007; 31: 1688-95.

29. Tournier A, Louis-Sylvestre J. Effect of the physical state of a food on subsequent intake in human subjects. Appetite 1991; 16: 17-24.
30. Levy RB, Claro RM e Monteiro CA. Aquisição de açúcar e perfil de macronutrientes na cesta de alimentos adquirida pelas famílias brasileiras (2002-2003). Cad Saúde Pública 2010; 26: 472-80.

31. Claro RM, Levy RB, Bandoni DH. Influência da renda sobre as despesas com alimentação fora do domicílio, no Brasil, 2002-2003. Cad Saúde Pública 2009; 25: 248996.

32. Naska A, Vasdekis VGS \& Trichopoulou A (2001) A preliminary assessment of the use of household budget survey data for the prediction of individual food consumption. Public Health Nutr 2001; 4(5B): 1159-65.

33. Becker W (2001) Comparability of household and individual food consumption data-evidence from Sweden. Public Health Nutr 2001; 4(5B): 1177-82.

Recebido em: 03/03/11 Versão final apresentada em: 03/09/11 Aprovado em: 17/10/11 